\title{
大气气溶胶液态水中二次有机气溶胶生成机制研究进展
}

\author{
肖瑶 ${ }^{1}$, 吴志军 ${ }^{1}$, 郭松 $^{1}$, 何凌燕 ${ }^{2}$, 黄晓锋 ${ }^{2}$, 胡敏 ${ }^{1 *}$ \\ 1. 北京大学环境科学与工程学院, 环境模拟与污染控制国家重点联合实验室, 北京 $100871 ;$ \\ 2. 北京大学深圳研究生院环境与能源学院, 城市人居环境科学与技术实验室, 深圳 518055 \\ *联系人, E-mail: minhu@pku.edu.cn
}

2020-06-15 收稿, 2020-08-09 修回, 2020-08-18 接受, 2020-08-21 网络版发表

国家自然科学基金(91844301，41977179，41875149)资助

摘要大气二次有机气溶胶是 $\mathrm{PM}_{2.5}$ 的主要组分, 具有重要的环境和气候效应. 在其主要生成途径中, 气溶胶液态水 中的液相生成是当前大气化学研究领域的前沿科学问题之一。液相二次有机气溶胶(aqueous secondary organic aerosol, aqSOA)前体物通过摄取进入湿气溶胶后, 参与气溶胶内部的液相反应, 生成有机硫、有机氮等aqSOA. 本 文对aqSOA前体物的摄取过程、生成的化学机制以及当前主要的研究手段进行详细总结, 并对该领域未来研究进 行展望. aqSOA前体物的摄取受到相对湿度、气溶胶水溶性等因素的影响，从而影响其进入气溶胶的后续反应. aqSOA生成的化学机制可分为自由基反应和非自由基反应，其中，自由基反应以 $\mathrm{OH}$ 自由基液相化学为主; 非自由 基反应则多为羰基化合物的液相反应。近年来，先进的离线和在线质谱技术的发展推动了对实际环境大气中 aq$\mathrm{SOA}$ 生成机制的认识, 并从分子层面识别出aqSOA产物, 但aqSOA生成的大气化学过程仍未明晰. 未来研究中, 开 发新的分析技术, 扩充aqSOA反应动力学数据库, 进一步完善模型模拟, 为aqSOA生成研究提供新思路.

关键词液相二次有机气溶胶, 前体物, 摄取, 液相反应, 生成机制, 分析技术

有机气溶胶(organic aerosol, OA)是 $\mathrm{PM}_{2.5}$ 的主要组 分, 占 $\mathrm{PM}_{2.5}$ 质量浓度的 $20 \% \sim 90 \%{ }^{[1]}$. OA包括污染源直接 排放的一次有机气溶胶(primary organic aerosol, POA)和 挥发性有机化合物(volatile organic compounds, VOCs)作 为气态前体物在大气中化学转化生成的二次有机气溶 胶(secondary organic aerosol, SOA). 早期的大气模型认 为, SOA的生成源于气相光化学过程生成的半挥发性产 物，通过气粒分配和凝结进人颗粒相的过程，即为气相 SOA(gas SOA). 根据这类模型模拟的气相SOA与观测 到的环境大气中SOA质量浓度相比，存在着严重的低 估 ${ }^{[2]}$, 模拟得到的OA约为观测值的 $10 \% \sim 60 \%{ }^{[3]}$. 而近年 来的研究发现液相过程生成SOA在大气中普遍存在, 将 环境大气云雾水和气溶胶液态水(aerosol liquid water, ALW)中反应生成的液相SOA(aqueous SOA, aqSOA)加
人大气模型中, 可明显改善对 SOA的模拟效果 ${ }^{[2,4]}$; 实验 室分析也证实，在潮解气溶胶中， $\alpha$-蒎烯和甲苯等前体 物的SOA生成产率均提高了 $10 \%$ 以上 ${ }^{[5]}$.

环境大气中的液相(aqueous phase)反应, 是以液态 水作为介质发生的化学反应. 而环境大气中的液相环 境包括：雨滴、云雾水和气溶胶中的液态水(也就是湿 气溶胶 ${ }^{[2,6,7]}$. 与气相反应不同, 许多物质在液相中的反 应途径会发生变化, 可能发生一些独特的化学反应, 如 质子化、水合作用、解离等. 气溶胶液态水即大气气 溶胶中存在的液态水分子，形成了湿气溶胶或湿颗粒 物的液相环境, 与云雾水有明显差异. 需要说明的是, 以往研究的表述中常常将气溶胶和颗粒物混淆，气溶 胶液态水实质上是颗粒物中的液态水，湿气溶胶也就 是湿颗粒物，本文使用约定俗成中更常用的气溶胶液

引用格式: 肖瑶, 吴志军, 郭松, 等. 大气气溶胶液态水中二次有机气溶胶生成机制研究进展. 科学通报, 2020, 65: 3118-3133 Xiao Y, Wu Z J, Guo S, et al. Formation mechanism of secondary organic aerosol in aerosol liquid water: A review (in Chinese). Chin Sci Bull, 2020, 65 3118-3133, doi: 10.1360/TB-2020-0713 
态水和湿气溶胶. 一方面, 湿气溶胶在大气中的寿命为 几天, 而云雾水仅几个小时且存在于特定环境中; 另一 方面，气溶胶液态水是大气气溶胶中普遍存在的组分, 其在全球湿气溶胶质量浓度中占比 $4 \%$ $85 \%$ (质量浓度 $\left.0.35 \sim 78 \mu \mathrm{g} \mathrm{m}^{-3}\right)^{[8]}$, 但气溶胶液态水含量远低于云雾水, 使得湿气溶胶中离子强度可能高达 $10 \mathrm{~mol} \mathrm{~L}^{-1}$ 以上 ${ }^{[9]}$. 这些差异表明气溶胶液态水中有较为独特的微观环境, 其中发生的大气化学过程与云雾液滴有所不同 ${ }^{[6,10]}$.

对aqSOA的研究, 以往多集中在云雾水体系中的 $\mathrm{SOA}$, 即在云雾水中氧化生成的有机物, 云雾水蒸发后 成为悬浮在大气中的颗粒物. 而最近的研究发现, 气溶 胶液态水中的有机物液相反应，可以生成高分子量有 机化合物(分子量可高达 1000 以上), 具有较高的氧碳比 (O/C可高达1 2), 进而影响颗粒物的吸湿性, 以及䨪和 云的生成 ${ }^{[2,7]}$; 生成的aqSOA是具有吸光性的有机物(棕 色碳, brown carbon), 关系到气溶胶的光吸收能力和辐 射平衡等气候效应 ${ }^{[7]}$. 本文关注的aqSOA是指气溶胶液 态水中液相反应生成的SOA, 下文均以aqSOA简称. 这 类aqSOA由于其独特的环境效应，以及尚不明晰的生 成机制, 成为大气化学领域研究的科学前沿和难点.

环境大气中气溶胶液态水的含量受到当地气象条 件、颗粒物吸湿性等因素的影响 ${ }^{[8]}$. 由于全球气候变 化, 地表蒸散速率的提高将增加大气中水汽的含量 ${ }^{[6]}$. 在我国, 随着大气污染防治对硫排放的控制, 颗粒物及 其中硫酸盐的质量浓度得以有效降低. 比如, 北京冬季 的亚微米颗粒物质量浓度，2017年比2014年下降了 $49.5 \%$ ，其中硫酸盐质量浓度降低 $64.1 \%{ }^{[11]}$. 而氨排放 量呈现增加趋势 ${ }^{[12]}$, 相比之下, $\mathrm{PM}_{2.5}$ 中硝酸盐的占比 有所增加 ${ }^{[13,14]}$. 硝酸盐的吸湿性较硫酸盐更强, 使得气 溶胶液态水的含量呈现出增加的趋势 ${ }^{[15]}$. 模型预计, 由 于排放源的变化, 截至2100年, 在我国华东地区，硝酸 盐成为二次无机盐中的主导成分，导致气溶胶液态水 含量进一步增加，而其中 $92 \%$ 的气溶胶液态水含量来 自人为硝酸铵颗粒物的贡献 ${ }^{[15,16]}$. 因此, 气溶胶液态水 中的aqSOA生成将变得更加重要, 成为持续改善空气 质量需要关注的科学问题.

本文综述aqSOA前体物的摄取、aqSOA生成的化 学机制以及当前主要的研究手段, 并对未来的工作进 行展望.

\section{1 aqSOA前体物的摄取}

气溶胶液态水中液相反应的反应物, 除了已存在
于湿气溶胶中的物质外，还有通过摄取进入湿气溶胶 中的痕量气体, 即aqSOA前体物. 这些痕量气体被湿气 溶胶摄取的过程是液相反应前体物到达颗粒物表面和 进人到颗粒物的过程, 是液相反应的第一步. 摄取过程 为, 前体物向气溶胶扩散, 被吸附在气溶胶表面上, 然 后被颗粒物吸收，最终溶解进入湿气溶胶 ${ }^{[17,18]}$, 如图 1 所示. 作为液相反应前体物的痕量气体被摄取进人湿 气溶胶后, 将发生一系列液相反应生成有机硫、有机 氮等aqSOA.

aqSOA前体物主要有两大类:（1）以自由基为主的 氧化剂, 如 $\mathrm{OH}$ 自由基、 $\mathrm{HO}_{2}$ 自由基、 $\mathrm{O}_{3}$ 、单线态氧 $\left(\mathrm{O}_{2}\right.$ $\left({ }^{1} \Delta_{\mathrm{g}}\right)$ 、、 $\mathrm{NO}_{3}$ 自由基、有机过氧自由基 $\left(\mathrm{RO}_{2}\right)^{\text {等 }}{ }^{[18]}$; 非自由基类的化合物, 主要是各种人为源和生物源挥 发性有机化合物, 以及它们在大气中的气相氧化产物, 如萜烯、醛酮酸类化合物、酚类化合物等 ${ }^{[9]}$.

对于aqSOA生成来说, SOA前体物的水溶性是非 常重要的, 而化合物在气相和液相之间的平衡由有效 亨利定律常数(effective Henry's law constant, $H_{\text {eff }}$ )决 定 ${ }^{[9,20]}$. 对于某一化合物, 其达到亨利定律平衡后, 可计 算其在液相的比例 $f_{\mathrm{aq}}$ :

$f_{\text {aq }}=\frac{R_{\mathrm{aq} / \mathrm{gas}}}{1+R_{\mathrm{aq} / \mathrm{gas}}}$,

$R_{\mathrm{aq} / \mathrm{gas}}=10^{-6} H_{\mathrm{eff}} R T L$,

其中, $R_{\mathrm{aq} / \mathrm{gas}}$ 是无量纲的比例, 表示该化合物在液相和气 相之间达到平衡后的浓度之比. 而在对 $R_{\mathrm{aq} / \mathrm{gas}}$ 的计算中, $10^{-6}$ 是单位换算系数; $H_{\mathrm{eff}}$ 是有效亨利定律常数 $\left(\mathrm{mol} \mathrm{L}{ }^{-1} \mathrm{kPa}^{-1}\right) ; R$ 是气体常数 $\left(8.314 \mathrm{~J} \mathrm{~K}^{-1} \mathrm{~mol}^{-1}\right) ; T$ 是

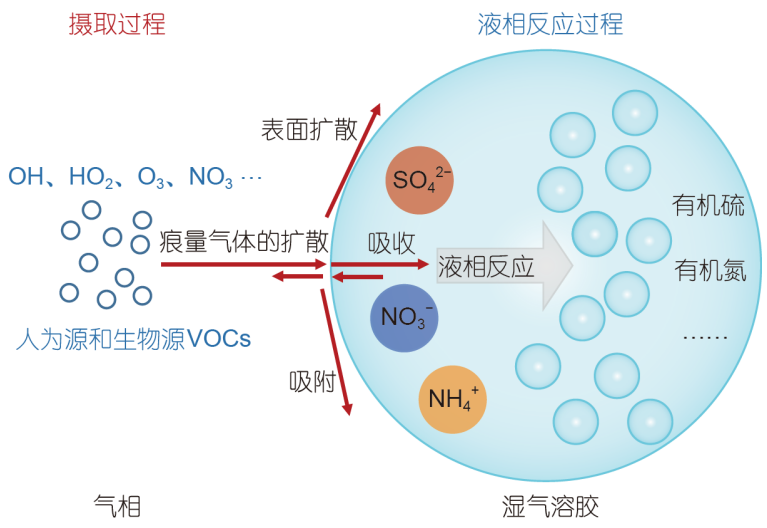

图 1 痕量气体在湿气溶胶上的摄取及后续的液相反应 ${ }^{[17,19]}$

Figure 1 Uptake of trace gases on a wet aerosol particle and subsequent aqueous-phase reaction ${ }^{[17,19]}$ 
温度 $(\mathrm{K}) ; L$ 是液态水质量浓度 $\left(\mathrm{g} \mathrm{m}^{-3}\right)$.

aqSOA前体物的气粒平衡是影响其在气溶胶上摄 取的重要因素. 从式(1)和(2)可以看出, 液态水质量浓度 $L$ 越高, 若 $H_{\mathrm{eff}}$ 不变, 化合物在液相中可分配的比例越高, 气溶胶液态水含量对于气体进入液相的过程有直接影 响. 但本文主要关注aqSOA前体物在湿气溶胶上的摄 取及气溶胶液态水对摄取的影响, 下面将从气态氧化 剂和VOCs两方面介绍摄取过程.

关于气溶胶液态水对氧化剂在有机气溶胶上摄取 的影响, 由于 $\mathrm{OH}$ 自由基是大气液相化学中最重要的氧 化剂 ${ }^{[6,21]}$, 故以 $\mathrm{OH}$ 自由基的摄取为例介绍. 由于水分子 能够形成较强的氢键, 具有相对较大的偶极矩 ${ }^{[22]}$, 水分 子可以被具有较高表面张力的表面有效吸收. 而被吸 附的水可以通过改变气溶胶的相态、黏度、活性表面 位点的性质等, 来影响 $\mathrm{OH}$ 自由基在有机气溶胶上的摄 取 ${ }^{[22]}$. 定量分析气溶胶液态水对 $\mathrm{OH}$ 自由基摄取的影响 是探究SOA液相生成的关键, 但是受到在实际环境大 气中测量技术的限制, 目前的研究很少, 多以相对湿度 作为指征, 探讨不同相对湿度下 $\mathrm{OH}$ 自由基摄取系数 (uptake coefficient)的变化.

表 $\mathrm{S} 1$ 总结了特定相对湿度下, $\mathrm{OH}$ 自由基在不同类 型有机气溶胶上的摄取系数. Slade和Knopf ${ }^{[25]}$ 研究了 生物质燃烧源有机气溶胶的 $\mathrm{OH}$ 自由基摄取中相对湿 度的作用. 分别用左旋葡聚糖(levoglucosan, LEV)和甲 基硝基邻苯二酚(methyl-nitrocatechol，MNC)代表生物 质燃烧源中的一次气溶胶和二次气溶胶, 发现LEV的 $\mathrm{OH}$ 自由基摄取系数 $\left(\gamma_{\mathrm{OH}}\right)$ 随着相对湿度的增加而增大, 而 $\mathrm{MNC}$ 的 $\gamma_{\mathrm{OH}}$ 则随着相对湿度增大而呈现下降趋势. LEV 的 $\gamma_{\mathrm{OH}}$ 值在高RH下更大, 可能源于气溶胶液态水对 气溶胶相态的控制, 而高RH可以降低颗粒物的秥度, 使得 $\mathrm{OH}$ 自由基和LEV 可以在更大程度上进行扩散混 合; 而 $\mathrm{MNC}$ 的 $\gamma_{\mathrm{OH}}$ 值在高 $\mathrm{RH}$ 下降低, 则可能是因为 $\mathrm{MNC}$ 的低水溶性 $\left(5.28 \mathrm{~g} \mathrm{~L}^{-1}\right)$ 导致水分子层聚集在表面, 通过 限制 $\mathrm{OH}$ 自由基进人气溶胶内部而抑制 $\mathrm{OH}$ 自由基的反 应性摄取. 或者说, 由于水分子对气溶胶表面活性位点 的竞争, $\mathrm{OH}$ 自由基的反应性摄取受到阻碍. Chan等 人 $^{[26]}$ 和Davies和Wilson ${ }^{[27]}$ 对氧化性有机气溶胶(oxygenated organic aerosol, OOA)中的丁二酸和柠檬酸对 $\mathrm{OH}$ 自由基的反应性摄取进行了研究. 丁二酸在 $\mathrm{RH}<10 \%$ 时 呈现为固态，而在 $R H$ 约为 $63 \%$ 时呈现为液态; 在高 $R H$ 下液滴中的丁二酸分子可以更快地从体相迁移到液滴 表面, 因而具有更高的 $\mathrm{OH}$ 自由基反应性. 而对于柠檬
酸，通过模型拟合得到的 $\gamma_{\mathrm{OH}}$ 值随着 RH的增加先升后 降. $\gamma_{\mathrm{OH}}$ 值的升高被认为是由于气溶胶相黏度的降低, 而随着RH的进一步增加, $\gamma_{\mathrm{OH}}$ 值的降低则源于颗粒物表 面水分子对 $\mathrm{OH}$ 自由基与柠檬酸碰撞的阻碍，即水分子 与 $\mathrm{OH}$ 自由基对气溶胶表面的活性位点存在竞争. 另外, 比较不同粒径柠檬酸的 $\gamma_{\mathrm{OH}}$ 值, 可看出粒径越小的柠檬 酸对 $\mathrm{OH}$ 自由基的反应性摄取越强，这可由小粒径颗粒 物的高比表面积来解释.

上面的研究对象均为单一有机物组分的颗粒物, 而实际大气气溶胶是有机气溶胶与无机气溶胶的复杂 混合物. 近年来有研究关注无机盐对有机物 $\mathrm{OH}$ 自由基 摄取的影响. Lam等人 ${ }^{[28]}$ 和Xu等人 ${ }^{[29]}$ 测定了3-甲基戊 二酸(3-methylglutaric acid, 3-MGA)和赤藓糖醇(erythritol) 在相对湿度 $85 \%$ (气溶胶呈现为液态)时的 $\gamma_{\mathrm{OH}}$ 值, 同 时测定了它们与硫酸铵(AS)混合后的气溶胶对 $\mathrm{OH}$ 自 由基的反应性摄取, 均发现硫酸铵无机盐的加人使得 有机气溶胶的 $\gamma_{\mathrm{OH}}$ 值降低. 这是由于无机盐的存在使得 气溶胶表面的有机物浓度比纯物质低, 有机物与 $\mathrm{OH}$ 自 由基的碰撞概率也随之下降，从而降低了对 $\mathrm{OH}$ 自由基 的反应性摄取. 另外, $\mathrm{Hu}$ 等人 ${ }^{[30]}$ 首次给出实际大气 $\mathrm{SOA}$ 的OH自由基摄取系数, 即美国东南部地区源于异戊二 烯环氧二醇的SOA(isoprene-epoxydiols-derived secondary organic aerosol, IEPOX-SOA)在不同相对湿度范围 下的 $\gamma_{\mathrm{OH}}$ 值，发现在 $\mathrm{RH}<90 \%$ 时， $\gamma_{\mathrm{OH}}$ 值变化不大，而 $\mathrm{RH}>90 \%$ 时， $\gamma_{\mathrm{OH}}$ 值明显增大. 认为在 $\mathrm{RH}>90 \%$ 时，气溶 胶有更高的液态水含量，使得气溶胶具有更大的表面 积，从而促进 $\mathrm{OH}$ 自由基向气溶胶相的摄取. $\mathrm{Li}$ 等人 ${ }^{[31]}$ 对 $\alpha$-蒎烯 $\mathrm{SOA}$ 的 $\mathrm{OH}$ 自由基摄取系数进行模型参数化的 结果显示, $\mathrm{RH}$ 约为 $30 \%$ 时的 $\gamma_{\mathrm{OH}}$ 值在 $0.1 \sim 1$ 之间, 而在 $\mathrm{RH}$ 约为 $90 \%$ 时, $\gamma_{\mathrm{OH}}$ 值在 $0.5 \sim 1$. Liu等人 ${ }^{[32]}$ 研究环已烯与 $\mathrm{OH}$ 自由基的光化学氧化时, 也认为更高的RH将提高 环已烯SOA的摄取系数, 促进SOA的老化.

从已有研究的结果来看, $\mathrm{OH}$ 自由基摄取随着 $\mathrm{RH}$ 的 变化受到有机气溶胶的粒径、水溶性 ${ }^{[25,27]}$ 等因素的影 响. 另外, 无机气溶胶与有机气溶胶的混合比例 ${ }^{[28,29]}$ 同 样会改变 $\gamma_{\mathrm{OH}}$ 值, 但当前研究多为对测定参数的描述, 对 于 $\mathrm{OH}$ 自由基在不同类型有机气溶胶上摄取的普遍规 律, 仍缺乏认识. 总地来说, 气溶胶液态水对 $\mathrm{OH}$ 自由基 摄取的影响可体现为：一方面，气溶胶呈现为液态，使 得气溶胶的黏度降低, 黏度与扩散系数直接相关, 气溶 胶中的物质可以更快速地扩散并进行充分地混合 ${ }^{[26,31]}$; 另一方面, 由于水会吸附在气溶胶上, 特别是一些疏水 
性有机气溶胶，水在气溶胶表面的吸附与 $\mathrm{OH}$ 自由基在 气溶胶上的摄取互相竞争气溶胶表面的活性位点, 从 而抑制 $\mathrm{OH}$ 自由基的摄取 ${ }^{[25,27]}$. 但当前研究多集中于单 组分有机气溶胶的实验室研究, 缺乏对实际环境大气 气溶胶 $\mathrm{OH}$ 自由基摄取受气溶胶液态水影响的直接 认识。

有关气溶胶液态水对VOCs在有机气溶胶摄取的 研究相对较少, 且主要集中在乙二醛和甲基乙二醛 上 $^{[33]}$ ，本文以甲基乙二醛为例介绍. 全球模型估算发 现，甲基乙二醛通过液相反应生成的 $\mathrm{SOA}$ 可达每年 1.5 8 $\mathrm{Tg} \mathrm{C}$, 占全球SOA每年生成量的 $1.1 \% \sim 6 \%{ }^{[33,34]}$. 表 $\mathrm{S} 2$ 总结了不同相对湿度下甲基乙二醛的摄取系数. Curry等人 ${ }^{[35]}$ 通过理论计算发现，随着相对湿度从 $50 \%$ 增至99\%，甲基乙二醛在硫酸铵-硝酸铵混合气溶胶上 的摄取系数 $\left(\gamma_{\mathrm{MGLY}}\right)$ 提高了 3 个数量级. 而De Haan等 人 $^{[36]}$ 在实验室测量得到甲基乙二醛在甘氨酸上的摄取 系数, $\gamma_{\mathrm{MGLY}}$ 值同样随着相对湿度增加而增大. 在硫酸 铵-硝酸铵体系(sulfate/nitrate/ammonium, SNA)和甘氨 酸气溶胶中, 随着相对湿度的增加, 气溶胶液态水含量 增大, SNA离子强度降低, 盐析效应有利于甲基乙二醛 进人SNA气溶胶; 而另一方面, 颗粒物黏度降低, 且湿 气溶胶表面积增大，均使得 $\gamma_{\mathrm{MGLY}}$ 值增大. 但Curry等 人 $^{[35]}$ 计算得到的 $\gamma_{M G L Y}$ 值远低于实验室结果，这主要是 因为理论计算中仅考虑甲基乙二醛进人气溶胶后与 $\mathrm{OH}$ 自由基的液相反应，而忽略了其他化学过程，使得 $\gamma_{\mathrm{MGLY}}$ 值被低估.

对于甲基乙二醛，已有的实验室研究和理论计算 均表明，气溶胶液态水的增加使其在湿气溶胶上的摄 取过程被促进. 但当前的研究仅限于某类特定气溶胶, 对实际大气气溶胶上的摄取不具有代表性，也缺乏合 理的参数化方案. 而对于其他VOCs的摄取, 更少有对 气溶胶液态水的考虑.

另外，表 $\mathrm{S} 1$ 和 $\mathrm{S} 2$ 中列举了摄取系数测定的研究方 法和主要的分析手段. 研究aqSOA前体物摄取的方法 主要为气溶胶流动管(aerosol flow tube, AFT)和烟雾箱 (smog chamber), 测量手段则主要是各种质谱技术，具 体可参见总结摄取系数测定的相关综述 ${ }^{[17,18]}$. 对于 aq$\mathrm{SOA}$ 前体物在湿气溶胶上的摄取，由于气溶胶液态水 对气溶胶离子强度、黏度、液态水分布及前体物扩散 速度等的改变, 使得摄取系数可能增大也可能降低. 当 前的研究对于不同前体物和不同种子气溶胶得到的摄 取系数差异较大，对应用于实际大气湿气溶胶的摄取，
难以总结出较完善的参数. 故需要加强对湿气溶胶上 前体物摄取的研究.

\section{2 aqSOA生成的化学机制}

气态前体物经过摄取进人湿气溶胶后，与气溶胶 中已存在的化学物质经过一系列的液相化学反应生成 aqSOA. 当前对aqSOA生成机制的研究还仅限于某几 种反应类型，一般按照是否有自由基参与aqSOA生成， 将aqSOA生成机制分为自由基化学过程和非自由基化 学过程 ${ }^{[2,6]}$.

\section{1 自由基液相反应}

自由基在液相中的反应机制主要有 3 种 ${ }^{[6]}$ : (1) 饱和 化合物的氢摘取; (2) 在不饱和化合物和芳香族化合物 碳碳双键上的亲电加成; (3) 电子转移. 由于 $\mathrm{OH}$ 自由基 在液相具有高反应性和非选择性，以 $\mathrm{OH}$ 自由基为例介 绍液相的自由基化学.

$\mathrm{OH}$ 自由基是大气液相最重要的氧化剂，可通过从 气相吸收进人液相，也可在液相中原位生成 ${ }^{[37]} . \mathrm{OH}$ 自 由基在液相的原位生成机制包括： $\mathrm{O}_{3}$ 进人液相中，与 $\mathrm{HO}_{2}$ 或其解离形式 $\mathrm{O}_{2}{ }^{-}$反应生成 $\mathrm{OH}$ 自由基 ${ }^{[38]}$; 一些无 机发色团, 如 $\mathrm{NO}_{3}{ }^{-} 、 \mathrm{NO}_{2}{ }^{-}$和 $\mathrm{H}_{2} \mathrm{O}_{2}$, 可直接在液相中光 解生成 $\mathrm{OH}$ 自由基 ${ }^{[39]}$; Fenton反应也可能生成 $\mathrm{OH}$ 自由 基 ${ }^{[40]}$; 另外，一些溶解的有机化合物也可能是液相 $\mathrm{OH}$ 自由基的重要来源，如部分有机发色团和有机过氧化 物 ${ }^{[41,42]}$. 受到测量技术的限制, $\mathrm{OH}$ 自由基的原位生成 速率是模拟自由基液相反应最大的不确定性来源之一. Arakaki等人 ${ }^{[43]}$ 总结了在不同大气环境液相条件下 $\mathrm{OH}$ 自由基的稳态浓度，虽然不同条件之间源和汇都存在 跨数量级的差异， $\mathrm{OH}$ 自由基稳态浓度基本处于 $0.5 \times$ $10^{15} \sim 7 \times 10^{15} \mathrm{~mol} / \mathrm{L}$ 的范围.

有机物的 $\mathrm{OH}$ 自由基液相反应研究较多. 与气相化 学相似, 如图2所示, $\mathrm{OH}$ 自由基可通过自由基加成与芳 香族化合物和不饱和脂肪族化合物反应. 没有自由基 加成的情况下， $\mathrm{OH}$ 自由基可通过快速的氢摘取过程与 液相的有机化合物反应, 随后往往进行 $\mathrm{O}_{2}$ 加成 ${ }^{[2]}$. 由于 这部分反应机制与气相 $\mathrm{OH}$ 自由基化学相似，气相化学 中关于 $\mathrm{OH}$ 自由基反应性的一般原则也适用于液相化 学, 下面重点介绍液相中独有的 $\mathrm{OH}$ 自由基反应.

\subsection{1 醛类向羧酸的有效转化}

醛类在液相中的水合可促进醛快速向羧酸转化, 比如早期的研究发现，甲酸在液相中通过甲醛的 $\mathrm{OH}$ 自 


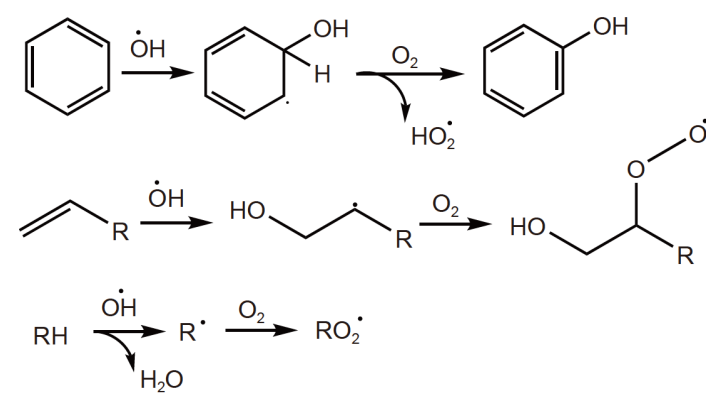

图 2 典型的OH自由基反应 ${ }^{[2]}$

Figure 2 Typical $\mathrm{OH}$ radical reactions ${ }^{[2]}$

由基氧化生成, 可提高液相的酸度 ${ }^{[9,44]}$.一般来说, 醛类 在液相中与其偕二醇平衡存在, $\mathrm{OH}$ 自由基可以从醛和 偕二醇上摘取氢原子, 生成烷基自由基, 氧分子与烷基 自由基反应生成过氧自由基，进一步快速解离生成羧 酸 ${ }^{[6,20]}$, 如图3所示. 大量实验室研究观察到乙二醛、甲 基乙二醛和乙醇醛在液相快速显著地生成有机酸, 而 气相化学缺乏羧酸的这类生成途径 ${ }^{[45,46]}$. 因此, 有机酸 尤其是草酸, 被认为是外场观测中液相化学的示踪

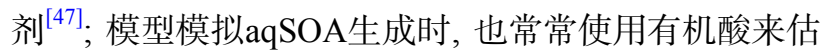
计aqSOA的产率和生成量 ${ }^{[2,24,48]}$.

\subsection{2 羧酸盐的快速 $\mathrm{OH}$ 自由基氧化}

羧酸盐在液相的 $\mathrm{OH}$ 自由基氧化比未解离的羧酸 更快, 故有机酸在液相的反应性表现出对 $\mathrm{pH}$ 的依赖

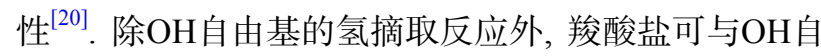
由基发生电子转移反应，生成 $\mathrm{OH}^{-}$和相应的有机自由 基 $^{[9,49,50]}$ (图4). 因而, 液相的酸度可能会改变有机酸的 寿命 ${ }^{[51]}$.

\subsection{3 自由基引发的低聚化反应}

$\mathrm{OH}$ 自由基还可引发自由基-自由基的低聚化反应, 即 $\mathrm{OH}$ 自由基通过氢摘取生成 2 个碳中心的有机自由基, 可通过自由基重组形成新的共价键，从而生成低聚 物 $^{[2,20]}$. 当乙二醛和甲基乙二醛在液相中的浓度为毫摩 尔或更高时，这种自由基-自由基重组在颗粒物液相中 变得重要 ${ }^{[2,24]}$. 芳香族化合物中也可观察到这类自由基 诱导的低聚化反应，如碳中心有机自由基与酚自由基 的重组 ${ }^{[23,52,53]}$.

另外, 实验室研究和大气环境颗粒物样品中观察到 的有机硫酸酯也可能是通过自由基化学生成的. Perri等 人 $^{[56]}$ 提出一种自由基重组机制, 其中烷基自由基和硫 酸盐自由基重组生成有机硫酸酯, 硫酸盐自由基源于 $\mathrm{OH}$ 自由基对硫酸、硫酸氢盐的氢摘取. 这种机制与自

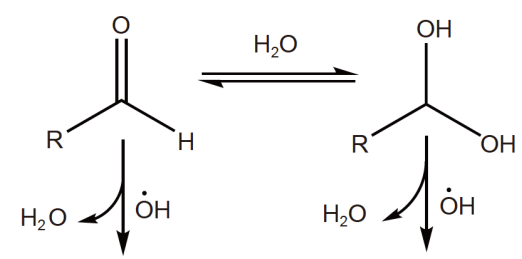<smiles>[R]C(=O)C=C([R])O</smiles><smiles>[R]C(=O)O[O]</smiles><smiles>CC(C)(O)OO</smiles><smiles>CC(O)=C(C)CO</smiles>

图 3 醛类在液相生成羧酸的机制 ${ }^{[6,20]}$

Figure 3 Mechanisms of carboxylic acid formation from aldehyde in the aqueous phase ${ }^{[6,20]}$<smiles></smiles>

图 4 羧酸盐在液相与 $\mathrm{OH}$ 自由基的电子转移反应 ${ }^{[20]}$

Figure 4 Charge transfer reaction of carboxylate with $\mathrm{OH}$ radical in the aqueous phase ${ }^{[20]}$

由基-自由基重组的低聚化反应类似(图5(a)). 而Schindelka等人 ${ }^{[57]}$ 则认为硫酸盐自由基在碳碳双键上的自由 基加成可生成有机硫酸酯(图5(b)). 有机硫酸酯被认为 是颗粒物液相化学反应的示踪剂 ${ }^{[48]}$.

表1对 $\mathrm{OH}$ 自由基液相化学生成aqSOA的实验条 件、反应动力学参数和产物进行了示例说明. 需要注 意的是, 这些研究得到的各反应物与 $\mathrm{OH}$ 自由基的二级 反应速率常数受到 $\mathrm{pH}$ 、温度等因素的影响，其对实际 大气反应动力学的适用性需要进一步验证. 除OH自由 基以外, 近年来的研究发现, 液相中存在其他的氧化剂, 如过氧自由基、单线态分子氧 ${ }^{1} \mathrm{O}_{2}$ * 、三重激发态的有 机化合物 ${ }^{3} \mathrm{C}^{*[6,53,62 \sim 64]}$ 等, 它们可能对SOA液相生成起 到重要作用. 如Smith等人 ${ }^{[53]}$ 发现生物质燃烧排放的芳 香族羰基化合物在光照下被激发到三重态，可以将酚 
(a)<smiles>CC(=O)C(C)OC(=O)C(C)(O)C(C)(O)C(=O)O</smiles>

(b)

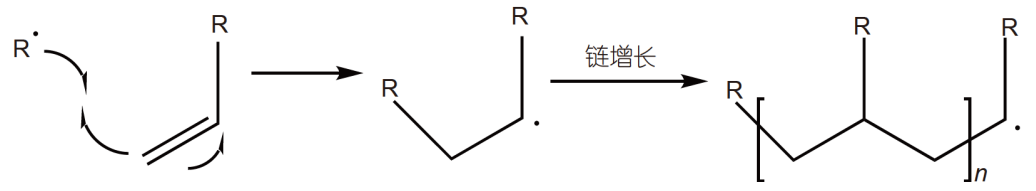

图 5 自由基引发的低聚化反应机制. (a) 自由基-自由基重组 ${ }^{[2,54]}$; (b) 双键上的链增长反应 ${ }^{[5]}$

Figure 5 Radical induced oligomerization mechanism. (a) Radical-radical recombination ${ }^{[2,54]}$; (b) radical propagation on double bonds ${ }^{[55]}$

表 $1 \mathrm{OH}$ 自由基液相反应生成aqSOA的实验条件、动力学参数和产物示例

Table 1 Examples of experimental conditions, kinetic data and products of $\mathrm{OH}$ radical aqueous reactions leading to aqSOA

\begin{tabular}{|c|c|c|c|c|c|}
\hline 反应物 & $\mathrm{pH}$ & 温度 $(\mathrm{K})$ & 二级反应速率常数 $\left(\mathrm{L} \mathrm{mol}^{-1} \mathrm{~s}^{-1}\right)$ & 产物 & 文献 \\
\hline 乙二醛 & 6 & 298 & $(9.2 \pm 0.5) \times 10^{8}$ & 乙二酸、乙醛酸、酒石酸等 & {$[58]$} \\
\hline 甲基乙二醛 & 6 & 298 & $(6.1 \pm 0.2) \times 10^{8}$ & 乙酸、丙酮酸等 & {$[50]$} \\
\hline \multirow{2}{*}{ 丙酮酸 } & 0 & 298 & $(3.2 \pm 0.6) \times 10^{8}$ & \multirow{2}{*}{ 乙酸、乙二酸等 } & \multirow{2}{*}[50]{} \\
\hline & 6 & 298 & $(7.1 \pm 1.8) \times 10^{8}$ & & \\
\hline \multirow{2}{*}{ 甲基乙烯基酮 } & 4 & 283 & $8 \times 10^{8}$ & $\begin{array}{c}\text { 乙二醛、甲基乙二醛、丙酮酸、乙酸、 } \\
\text { 乙二酸、高分子量化合物等 }\end{array}$ & [59] \\
\hline & 6 & 298 & $(7.3 \pm 0.5) \times 10^{9}$ & $\begin{array}{c}\text { 甲基乙二醛、乙醇醛、丙酮酸、 } \\
\text { 多官能团 } \mathrm{C}_{4} \text { 化合物等 }\end{array}$ & [60] \\
\hline 甲基丙烯醛 & 6 & 298 & $(9.4 \pm 0.7) \times 10^{9}$ & $\begin{array}{c}\text { 甲基乙二醛、羟基丙酮、乙醇醛、 } \\
\text { 丙酮酸、多官能团 } \mathrm{C}_{4} \text { 化合物等 }\end{array}$ & [60] \\
\hline \multirow{2}{*}{ 4-硝基酚 } & 5.2 & 298 & $4.1 \times 10^{9}$ & & \multirow{2}{*}[61]{} \\
\hline & 9.2 & 298 & $8.7 \times 10^{9}$ & & \\
\hline
\end{tabular}

类的氢原子摘取形成酚类自由基，进一步通过自由基 重组生成低聚物。Kaur等人 ${ }^{[64]}$ 测定了三重激发态的苯 甲酮与 17 种水溶性 $\mathrm{C}_{3} \sim \mathrm{C}_{6}$ 烯烃液相反应的速率常数. 但 对于这些氧化剂液相反应的研究主要集中在反应速 率、产率等的测定上, 生成机制尚不清楚.

\section{2 非自由基液相反应}

近年来，非自由基液相反应备受关注. 一方面，非 自由基液相反应是SOA生成的潜在途径，但其贡献在 当前的大气模型中往往被低估; 另一方面, 非自由基液 相反应可生成高分子量的产物，解释了大气中观测到 的一些高分子量有机化合物 ${ }^{[2,6]}$. 目前的研究多为对反 应产物的测定，在生成机制和反应动力学上的认知 不足.

\subsection{1 半缩醛生成}

半缩醛的生成通常需要醇作为亲核试剂与羰基化 合物反应. 简单来说, 羰基被 $\mathrm{H}^{+}$质子化后, 羟基攻击质 子化的羰基从而生成半缩醛(图6 $)^{[6,42]}$. 对于一些水合平 衡常数较大的醛类化合物来说，如乙二醛、甲基乙二 醛、甲醛, 这些醛类的偕二醇可充当亲核试剂, 实现自 身低聚化 ${ }^{[65 \sim 67]}$. 另外, 糖类是含有多个羟基的醛酮类化 合物，也可生成半缩醛，如葡萄糖的直链异构体，其分 支两端分别有差基和醛基，可通过分子内半缩醛的生 成产生环状化合物 ${ }^{[42]}$. 已有研究发现, 在左旋葡聚糖的 $\mathrm{OH}$ 自由基氧化实验中，检测到左旋葡聚糖通过半缩醛 生成低聚化生成的反应中间产物 ${ }^{[68,69]}$.

\section{2 .2 羟醛缩合}

差醛缩合则需要烯醇作为亲核试剂，烯醇与其羰 


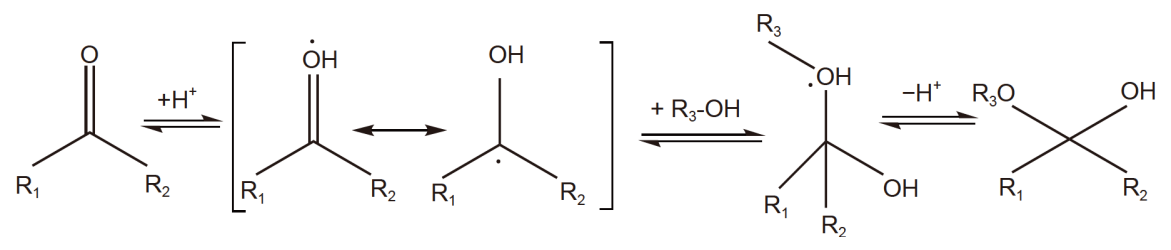

图 6 液相中半缩醛的生成 ${ }^{[42,65]}$

Figure 6 Hemiacetal formation in the aqueous phase ${ }^{[42,65]}$

基化合物形式的醛类在液相中平衡存在. 烯醇向醛进 行亲核加成首先生成羟醛中间体，再通过脱水生成最 终产物(图7) ${ }^{[6]}$. 对于不易生成偕二醇的醛酮类化合物, 差醛缩合是其低聚化的主要反应机理 ${ }^{[70,71]}$. 大量研究 已发现大气中 $\alpha$-二羰基化合物(如乙二醛、甲基乙二 醛)的半缩醛和羟醛缩合物的混合产物 ${ }^{[65,66,72]}$.

需要注意的是, 尽管半缩醛生成和羟醛缩合是非 自由基条件下低聚化的重要反应机制，但两者的反应 产物存在本质上的差异. 由于差醛缩合中有脱水步骤, 差醛缩合物的氧化程度更低, 而半缩醛则保留了所有 的氧原子. 脱水步骤在羟醛缩合物上产生双键, 形成可 吸光的 $\pi$ 键共轭体系, 从而可能贡献大气中的有机发色 团. Noziere等人 ${ }^{[70,73,74]}$ 提出羟醛缩合产物与大气棕色 碳之间的联系. 同时有研究发现柠檬烯SOA提取物的 差醛缩合可产生高度共轭的强吸光性低聚物 ${ }^{[75]}$.

\subsection{3 亚胺的生成}

含有孤对电子的还原态氮化合物, 如氨、氨基 酸、伯胺, 可与羰基化合物亲核加成生成亚胺(包括咪
唑类化合物 $)^{[2]}$. 还原态氮化合物上的胺基亲核攻击羰 基, 生成醇胺类中间产物, 随后脱水生成含有碳氮双键 的亚胺(图8). 亚胺在液相中后续反应可以生成含氮有 机发色团, 是棕色碳的重要来源 ${ }^{[76]}$. 实验室研究已观察 到，乙二醛、甲基乙二醛可通过一系列加成反应生成 咪唑及其衍生物 ${ }^{[77,78]}$.

表2举例说明了几个非自由基液相化学生成aq$\mathrm{SOA}$ 的研究. 非自由基反应的速率常数同样受到催化 条件、 $\mathrm{pH}$ 、温度等因素的影响, 这些动力学参数在实 际外场观测和模型模拟中的应用仍较为有限.

\subsection{4 其他的非自由基液相反应}

在液相中普遍存在的酸类，可发生非自由基液相 反应生成酸酐 ${ }^{[2]}$. 1 个羧酸的差基作为亲核试剂攻击另 1 个羧酸的羰基, 随后进行脱水, 即生成酸酐. 比如, 有 研究发现烟雾箱中碳氢化合物臭氧化实验可生成酸酤 ${ }^{\text {产物 }}{ }^{[80]}$. 另外, 除自由基反应外, 硫酸酯也可通过醇类 和硫酸的酸催化反应生成, 如烟雾箱中乙二醛与硫酸 的液相反应 ${ }^{[81]}$; 烟雾箱实验中萜烯在硫酸酸化的种子

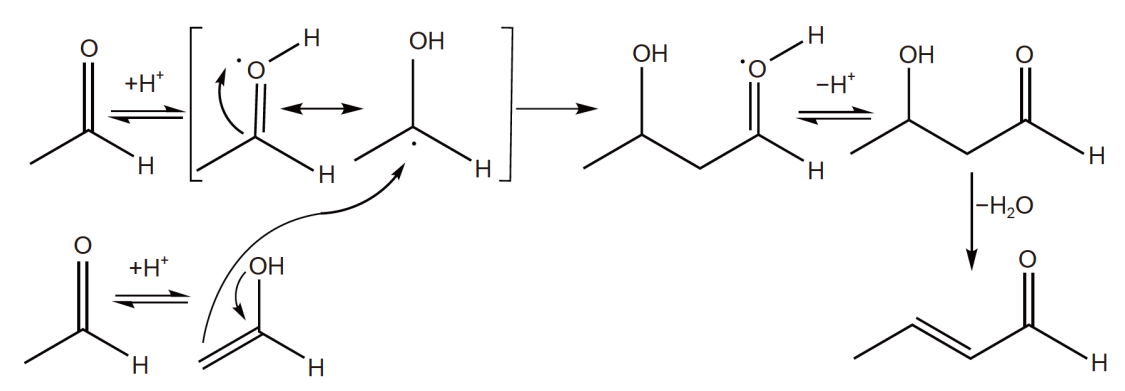

图 7 液相中的羟醛缩合 ${ }^{[6]}$

Figure 7 Aldol condensation in the aqueous phase ${ }^{[6]}$

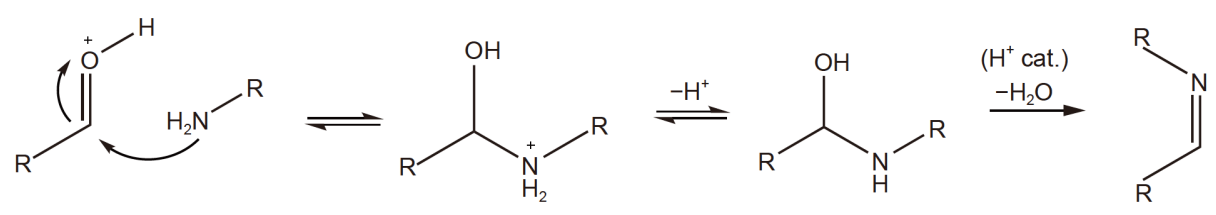

图 8 液相亚胺的生成 ${ }^{[2,6,42]}$

Figure 8 Imine formation in the aqueous phase ${ }^{[2,6,42]}$ 
表 2 非自由基液相反应生成aqSOA的实验条件、动力学参数和产物示例

Table 2 Examples of experimental conditions, kinetic data and products of non-radical aqueous reactions leading to aqSOA

\begin{tabular}{|c|c|c|c|c|c|}
\hline 有机物 & 主要反应物/条件 & $\mathrm{pH}$ & 反应速率常数 $\left(\mathrm{L} \mathrm{mol}^{-1} \mathrm{~s}^{-1}\right)$ & 产物 & 文献 \\
\hline 乙醛 & \multirow{5}{*}{$\begin{array}{c}\mathrm{H}_{2} \mathrm{SO}_{4} \text { 酸(质量分数 } 80 \% \text { ) } \\
\text { 催化体系 }\end{array}$} & & $\sim 0.005$ & \multirow{5}{*}{$\begin{array}{c}\text { 脱水羟醛缩合产物, 如丁烯醛、 } \\
\text { 2-乙基-已烯醛等 }\end{array}$} & \multirow{5}{*}[71]{} \\
\hline 丙醛 & & & $\sim 0.15$ & & \\
\hline 正丁醛 & & & $\sim 0.17$ & & \\
\hline 正戊醛 & & & $\sim 0.065$ & & \\
\hline 正己醛 & & & $\sim 0.035$ & & \\
\hline 甲基乙二醛 & $\left(\mathrm{NH}_{4}\right)_{2} \mathrm{SO}_{4}$ & 2 & $8.3 \times 10^{-8}$ & 低聚氨基化合物、有机硫酸酯等 & {$[65]$} \\
\hline \multirow{4}{*}{ 甲基乙二醛 } & 精氨酸 & & $10^{0.422 \mathrm{pH}-4.09}$ & \multirow{4}{*}{ 甲基咪唑等 } & \multirow{4}{*}{ [79] } \\
\hline & 甘氨酸 & & $10^{0.262 \mathrm{pH}-3.40}$ & & \\
\hline & 丝氨酸 & 200 & $10^{0.421 \mathrm{pH}-3.91}$ & & \\
\hline & 甲胺 & & $10^{0.334 \mathrm{pH}-3.22}$ & & \\
\hline
\end{tabular}

颗粒物上光氧化生成的硫酸酯产物 ${ }^{[82]}$. 当前对非自由 基液相反应生成aqSOA取得了一些进展，但这些反应 机制在大气化学模型框架中的应用仍很有限, 且大量 研究主要是实验室模拟结果, 如何与环境大气观测结 果相关联, 有待进一步的探索.

\section{3 aqSOA生成的研究手段}

在环境大气中直接观测 SOA液相化学过程较为困 难, 因为实际大气中有机物种类繁多且浓度较低, 各种 化学过程相互影响. 因而, 实验室模拟是目前常用的研 究手段, 对aqSOA的实验室研究方法包括体相溶液 (bulk solution)模拟和反应箱(reaction chamber) 实验 ${ }^{[83]}$.

体相溶液模拟是指在玻璃反应器(一般为几十到几 百毫升)中加人一定量的前体物溶液、氧化剂和其他需 要的物种进行暗反应或有光照条件下的反应, 观察溶液 反应生成产物的产率、化学性质等, 来模拟云雾水和气 溶胶液态水中相似浓度条件下的反应 ${ }^{[83,84]}$. 实际大气液 相中有机物的浓度水平变化较大, 以往的研究认为大气 中有机物在云雾水中的浓度约为 $0.01 \sim 1 \mathrm{mmol}$, 而在气 溶胶液态水中为 $0.01 \sim 10 \mathrm{~mol}^{[55,84]}$, 在体相溶液实验中 一般选用拟研究前体物的高纯度物质溶解于超纯水 $\left(\geqslant 18.2 \mathrm{M} \Omega \mathrm{cm}\right.$, Millipore)中 ${ }^{[52]}$. 另外, 大量外场观测表 明气溶胶具有从弱碱性到强酸性的 $\mathrm{pH}^{[6,85,86]}$ ，在进行体 相溶液实验时, 通常使用硫酸来调节溶液 $\mathrm{pH}^{[84]}$. 模拟液 相OH自由基反应时常用的 $\mathrm{OH}$ 自由基来源为 $\mathrm{H}_{2} \mathrm{O}_{2}$, 除使 用 $\mathrm{OH}$ 自由基作为氧化剂外，近年来的研究也关注过渡 金属离子 $\left(\right.$ 如 $\left.\mathrm{Fe}^{3+} 、 \mathrm{Mn}^{2+}\right) 、{ }^{3} \mathrm{C}^{*} 、{ }^{1} \mathrm{O}_{2}{ }^{*}{ }^{[53,84,87,88]}$ 等作为 $\mathrm{aq}-$
SOA生成研究的氧化剂. 在大气光照条件下通常使用氙 灯或采灯来模拟，而暗反应则是将用铝䈃包裹的体相溶 液放置在同样的环境下进行 ${ }^{[53,55,89]}$. 早期的aqSOA实验 室研究多为体相溶液模拟, 对多种水溶性有机物种进行 体相溶液实验, 包括小分子量羰基化合物、异龙二烯及 其氧化产物和酚类化合物 ${ }^{[2,53,55,90]}$ 等.

体相溶液实验较为简单方便, 但其问题在于, 体相 溶液不是真实的分散的颗粒物体系，以及体相溶液中 液态水的浓度为 $10^{3} \mathrm{~g} \mathrm{~m}^{-3}$ (按纯水的密度算), 而实际大 气即使是云滴中, 液态水的浓度也仅在 $1 \mathrm{~g} \mathrm{~m}^{-3}$ 的数量 级 ${ }^{[42,83]}$. 另外, 在体相溶液体系, 氧化剂一般直接加人 液相中，而实际大气中的气相氧化剂需要通过气粒分 配进人液相, 如气相 $\mathrm{OH}$ 自由基. 这些差异使得体相溶 液模拟较难以与实际大气中的化学过程进行对比. 如 Daumit等人 ${ }^{[83]}$ 比较了多元醇在体相溶液体系和亚微米 颗粒物烟雾箱实验中的液相化学的差异, 发现由于烟 雾箱中液态水含量远低于体相溶液中，在凝结相中的 $\mathrm{SOA}$ 氧化产物产率也比体相溶液低. 这主要是因为多 元醇液相氧化的中间产物在烟雾箱中更容易分配进人 气相, 这一过程与进一步的液相氧化过程竞争, 也说明 体相溶液体系对实际大气aqSOA的模拟存在较大的局 限性. 而反应箱实验能够模拟更接近实际大气的化学 演化过程, 与外场观测有更好的可比性.

反应箱实验是使用一定体积的反应箱来模拟二次 有机气溶胶生成及演化机制，近年来越来越多地应用 于aqSOA生成的研究中. 反应箱主要分为烟雾箱和流 动管(flow tube $)^{[91]}$. 烟雾箱和流动管的差异在于反应箱 
的体积，烟雾箱一般在数立方分米到数百立方米之间， 而流动管的体积较小, 通常为 $<1 \mathrm{~m}^{3}$. 从体积上的差异 可以看出，流动管较容易搭建，而烟雾箱则不易移动; 另一方面，烟雾箱一般可模拟实际大气中几个小时甚 至几天的演化过程, 而流动管中往往提供较高的氧化 环境加速前体物的老化，从而实现在较短的实验时间 (分钟到小时量级)模拟实际大气中几天的氧化过 程 ${ }^{[91,92]}$. 烟雾箱和流动管在实验流程上类似, 即在反应 箱中加人相应的前体物、气溶胶种子、氧化剂等, 调 节相对湿度等条件, 以达到高离子强度的气溶胶液相

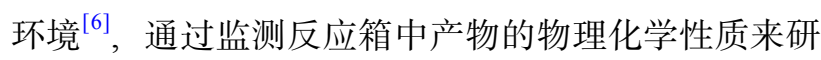
究有机气溶胶液相过程的演化 ${ }^{[93]}$. 比如, Surratt等人 ${ }^{[82]}$ 和Iinuma等人 ${ }^{[94]}$ 通过烟雾箱实验发现了通过非自由基 反应生成的有机硫酸酯, 而Schindelka等人 ${ }^{[57]}$ 则在烟雾 箱实验中阐明有机硫酸酯的自由基反应途径. 总地来 说, 反应箱实验是当前研究SOA夜相化学的主要手段.

在对 SOA夜相化学的研究上, 很重要的一方面在 于分析技术的进展，尤其是质谱技术的发展 ${ }^{[6]}$. 近年来 较常用于aqSOA研究的质谱技术包括: (1) 电喷雾电离 质谱(electrospray ionization-mass spectrometry, ESI$\mathrm{MS}$ ); (2) 傅里叶变换离子回旋共振质谱(Fourier transform ion cyclotron resonance electrospray ionization mass spectrometry, FTICR-MS); (3) 化学离子化质谱(chemical ionization mass spectrometry, CIMS); (4) 电喷雾萃取 电离质谱(extractive electrospray ionization-mass spectrometry, EESI-MS)如表3所示. 其中, ESI-MS和FTICRMS为离线测量, CIMS和EESI-MS技术则可实现在线测 量. ESI-MS技术通过使样品溶液喷雾在强电场下生成 带电液滴，进人质谱后对大分子进行检测，ESI-MS广 泛应用于对有机硫酸酯的检测中 ${ }^{[05,96]}$. FTICR-MS技术 则通过傅里叶变换得到高强磁场中带电离子回旋运动 频率, 从而获得精确的分子量和元素组成信息. 已有一 些研究使用该技术来识别雾水和气溶胶中的有机 物 $^{[97,98]}$. CIMS 与气体和气溶胶滤膜进样口(filter inlet for gas and aerosols, FIGAERO)相结合, 通过使用不同 的试剂离子作为软电离源, 实现对气溶胶中有机物的 实时测量 ${ }^{[99,100]}$. 而EESI-MS技术则通过对进样口和电 离源的设计, 使得气溶胶样品可直接进样与电喷雾产 生的带电液滴喷雾混合, 进人质谱进行在线分析 ${ }^{[101]}$, EESI-MS最早应用于Doezema等人 ${ }^{[102]}$ 检测 $\alpha$-蒎烯与 $\mathrm{O}_{3}$ 反应生成SOA中的有机物. 总地来说, 这些质谱技术可 实现对aqSOA中复杂有机物分子水平的准确识别, 但
对具体物种的定量仍有较大的难度，取决于是否存在 标准品等因素; 另外, 相较于实验室样品, 环境大气气 溶胶的实时测量, 由于成分更为复杂, 对于质谱数据的 分析难度也更大.

除实验室模拟外, aqSOA的研究也包括外场观测 和模型模拟. 就外场观测而言, 目前的研究技术很难从 复杂的大气环境中直接测量得到aqSOA. 大多数观测 选择高相对湿度或者雾天等特殊的气象条件进行, 但 难以确定是雾水还是气溶胶液态水中生成的 $\mathrm{SOA}^{[7]}$; 或者使用受体模型源解析等手段来估算外场观测中生 成的aqSOA. 但总地来说, 外场观测对aqSOA是否是在 气溶胶液态水中生成, 判断较为模糊, 一般笼统地称为 液相SOA, 或者仅仅表明有液相过程发生. 比如, $\mathrm{Xu}$ 等 人 $^{[105]}$ 使用气溶胶质谱仪(AMS)对北京夏秋冬3个季节 进行观测, 结合正矩阵因子分子(PMF)对有机气溶胶进 行源解析, 发现随着RH增加, OA中高氧化态氧化性有 机气溶胶(MO-OOA)的质量占比增加, 同时, SOA的氧 碳比随着RH升高明显增大，综合这些因素认为，液相 过程对于高氧化态SOA的生成很重要. Xu等人 ${ }^{[106]}$ 在美 国东南部外场观测中发现，异戊二烯来源的有机物与 硫酸盐具有良好的相关性, 并综合实验室研究结果, 认 为异龙二烯在气相氧化生成IEPOX后, 进人气溶胶液 相进行一系列液相反应. Gilardoni等人 ${ }^{[107]}$ 在意大利的 外场观测中, 用AMS-PMF解析出液相SOA, 并通过综 合分析其谱图特征等, 认为液相SOA源于生物质燃烧 排放过程. 如何在原位观测中直接识别出气溶胶液态 水中生成的 SOA, 仍是目前外场观测的难点.

而在模型模拟方面，早期的SOA液相化学模拟主 要关注云水化学. 近年来, 一些模型开始关注气溶胶液 态水中的SOA生成, 但部分模型直接把云水化学模型 扩展到气溶胶液相中, 使用的动力学参数并不完全适 用 ${ }^{[7]}$. 对aqSOA的模拟，大多仍集中在乙二醛和甲基乙 二醛等小分子羰基化合物上. 如Ervens和Volkamer ${ }^{[10]}$ 为 乙二醛生成aqSOA开发了一套动力学模型框架, 这一方 法被用于盒子模型中研究墨西哥城乙二醛生成的

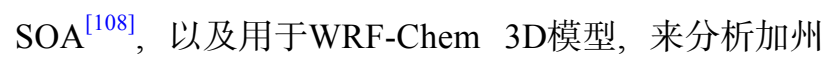
CARES/CalNex观测的数据 ${ }^{[109]}$. 而一些模拟方法只是 在原有的大气化学模型上增加新的aqSOA生成途径, 如Pye等人 ${ }^{[110]}$ 通过在CMAQ模型上加人异戊二烯氧化 产物的液相化学过程, 改善了对美国东南部OA的模拟. 另外, McNeill研究组 ${ }^{[48]}$ 开发了一个光化学盒子模型 GAMMA(gas-aerosol model for mechanism analysis), 该 
表 3 质谱技术用于aqSOA识别的近期研究示例

Table 3 Recent examples of mass spectrometry for aqSOA identification research

\begin{tabular}{|c|c|c|c|}
\hline 样品/实验 & 质谱技术 & 测量物种 & 文献 \\
\hline 上海 $\mathrm{PM}_{2.5}$ 膜样品 & UHPLC-Orbitrap MS & 定量识别14种有机硫酸酯, 如 $\mathrm{C}_{4} \mathrm{H}_{7} \mathrm{O}_{7} \mathrm{~S}^{-}(m / z 198.9918)$ & [96] \\
\hline 北京总悬浮颗粒物膜样品 & FTICR-MS & $\begin{array}{c}\text { 分子水平上识别超过 } 8000 \text { 种有机硫酸酯, 包括 } \\
500 \sim 800 \mathrm{Da} \text { 的高分子量有机硫酸酯 }\end{array}$ & [98] \\
\hline$\alpha$-蒎烯臭氧分解生成的 $\mathrm{SOA}$ 与 $\mathrm{OH}$ 的液相反应 & Aerosol-ToF-CIMS & 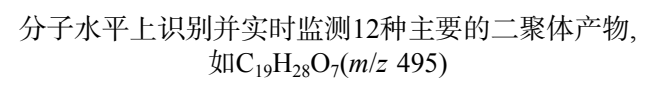 & [103] \\
\hline$\alpha$-蒎烯氧化实验 & EESI-MS & $\begin{array}{c}\text { 分子水平上识别多种 } \alpha \text {-蒎烯氧化产物, 如 } \mathrm{C}_{17} \mathrm{H}_{26} \mathrm{O}_{8}(m / z \\
358.1628)\end{array}$ & [104] \\
\hline
\end{tabular}

模型耦合了气相和气溶胶液相化学, 能够用于模拟液 态气溶胶中SOA生成, 包括有机硫酸酯的生成. 在此基 础上, GAMMA的简化版本simpleGAMMA ${ }^{[111]}$ 通过只追 踪GAMMA模型中最重要的几种液相物种来达到近似 的模拟效果，可以与更大尺度的大气化学模型耦合使 用. 总地来说, 对aqSOA的模拟依赖于实验室对aqSOA 生成机制的研究以及反应动力学参数的精确测量. aqSOA模拟相较于gasSOA的不完善主要源于重要机制及 模型参数的缺失, 使得aqSOA模型的进一步发展受限.

\section{4 总结与展望}

随着对SOA生成途径认识的深人，aqSOA成为近 年来的研究热点. 大量实验室研究对不同前体物在液 相中生成SOA的动力学参数和反应机制进行了细致的 分析，但目前对于 aqSOA生成机制的了解仍较为局限, 很多关键参数具有极大的不确定性甚至暂时缺乏，因 而难以对大气中aqSOA的贡献进行准确的定量. 另一 方面，近年来，一些物种如羟甲基磺酸盐(hydroxymethanesulfonate, HMS)在污染大气中的液相生成受到 关注 ${ }^{[112,113]}$, 在华北平原冬季䨪污染中, HMS在颗粒有 机物的质量占比可高达 $15 \%{ }^{[113]}$, 对霧污染有重要贡献, 但其生成机制仍不明晰; 在aqSOA物化性质的研究中 发现, 不同产物可能有明显的吸光性, 或者显著的吸湿 性, 从而影响气溶胶的光学性质和气候效应, 但对于aqSOA环境效应的认识仍局限于某几类化合物. 基于此, 对未来aqSOA的研究提出以下展望.

(1) 建立aqSOA前体物摄取的参数化方案. 过去对 于气态前体物在气溶胶上摄取的研究, 多为在干气溶 胶上的摄取, 而对湿气溶胶上摄取的认识不足. 目前大 多数关于摄取过程的研究仅考虑了相对湿度的影响, 且使用成分较为单一的气溶胶，难以代表实际大气气 溶胶, 缺乏气溶胶液态水含量、不同化学组分、 $\mathrm{pH}$ 等
因素对摄取影响的评估. 另外, 不同的实验室研究由于 方法的差异，得到的摄取系数可能存在几个数量级的 差异. 未来需要对摄取进行更深人的研究, 建立起可代 表实际大气并应用于模型中的参数化方案.

(2) aqSOA生成机制及反应动力学研究. 在aqSOA 生成的实验室研究中, 对生成机制的研究往往是基于 单一组分气溶胶和氧化剂的反应，对 $\mathrm{OH}$ 自由基、 $\mathrm{O}_{3}$ 等 之外的氧化剂的认知较少. 近年来对三重激发态化合 物作为氧化剂的关注增加, 但主要集中在生物源气溶 胶上. 而另一类aqSOA生成研究主要关注生成SOA的 产率, 但对于生成的各类产物缺乏认识, 也不清楚产物 各组分占比及反应途径. aqSOA生成机制和反应动力 学参数的有机结合，有利于对实际大气 aqSOA生成的 估计.

(3) 发展aqSOA分析技术. 目前对aqSOA的分析主 要采用质谱技术. 质谱技术可以很好地实现对有机物 分子层面的识别，但对物种分子结构的认识和定量等 方面较为有限. 另外, 在研究aqSOA时, 往往需要气溶 胶液态水含量、 $\mathrm{pH}$ 等相关信息. 大多数研究所采用模 型结合化学组分等信息估算这些参数，由于缺乏相关 的原位直接测量手段, 对气溶胶液相性质的认识有较 大的不确定性. 同时，在实际大气外场观测中，难以对 aqSOA生成进行直接测量或表征, 需要寻找具有示踪 性的物质, 来定量aqSOA的贡献.

(4) 改善大气模型中SOA的模拟效果. 由于对aq$\mathrm{SOA}$ 生成研究的局限性, 目前的模型研究中, 对SOA生 成的考虑多为云水化学，主要关注乙二醛和甲基乙二 醛的液相化学, 或者有机硫酸酯等特定物种, 缺乏对 aqSOA总量的估算. 随着分析技术的发现，对aqSOA生成 机制及反应动力学研究的进一步完善, 实验室获得的 各项参数可加人大气模型中，并能够用于评估aqSOA 的环境效应. 


\section{参考文献}

1 Jimenez J L, Canagaratna M R, Donahue N M, et al. Evolution of organic aerosols in the atmosphere. Science, 2009, 326: 1525-1529

2 Lim Y B, Tan Y, Perri M J, et al. Aqueous chemistry and its role in secondary organic aerosol (SOA) formation. Atmos Chem Phys, 2010, 10: $10521-10539$

3 Heald C L, Coe H, Jimenez J L, et al. Exploring the vertical profile of atmospheric organic aerosol: Comparing 17 aircraft field campaigns with a global model. Atmos Chem Phys, 2011, 11: 12673-12696

4 McNeill V F. Aqueous organic chemistry in the atmosphere: Sources and chemical processing of organic aerosols. Environ Sci Technol, 2015, 49: $1237-1244$

5 Faust J A, Wong J P S, Lee A K Y, et al. Role of aerosol liquid water in secondary organic aerosol formation from volatile organic compounds Environ Sci Technol, 2017, 51: 1405-1413

6 Herrmann H, Schaefer T, Tilgner A, et al. Tropospheric aqueous-phase chemistry: Kinetics, mechanisms, and its coupling to a changing gas phase. Chem Rev, 2015, 115: 4259-4334

7 Ervens B, Turpin B J, Weber R J. Secondary organic aerosol formation in cloud droplets and aqueous particles (aqSOA): A review of laboratory, field and model studies. Atmos Chem Phys, 2011, 11: 11069-11102

8 Nguyen T K V, Zhang Q, Jimenez J L, et al. Liquid water: Ubiquitous contributor to aerosol mass. Environ Sci Technol Lett, 2016, 3: 257-263

9 John H S, Spyros N P. Atmospheric Chemistry and Physics: From Air Pollution to Climate Change. 3rd ed. Hoboken: Wiley, 2016

10 Ervens B, Volkamer R. Glyoxal processing by aerosol multiphase chemistry: Towards a kinetic modeling framework of secondary organic aerosol formation in aqueous particles. Atmos Chem Phys, 2010, 10: 8219-8244

11 Li H, Cheng J, Zhang Q, et al. Rapid transition in winter aerosol composition in Beijing from 2014 to 2017: Response to clean air actions. Atmos Chem Phys, 2019, 19: 11485-11499

12 Stocker T F, Qin D, Plattner G K, et al. Climate Change 2013: The Physical Science Basis. In: Working Group I Contribution to the Fifth Assessment Report of the Intergovernmental Panel on Climate Change. New York: Cambridge University Press, 2014

13 Bauer S E, Koch D, Unger N, et al. Nitrate aerosols today and in 2030: A global simulation including aerosols and tropospheric ozone. Atmos Chem Phys, 2007, 7: 5043-5059

14 Pye $\mathrm{H} \mathrm{O}$, Liao H, Wu S, et al. Effect of changes in climate and emissions on future sulfate-nitrate-ammonium aerosol levels in the United States. J Geophys Res-Atmos, 2009, 114: D01205

15 Hodas N, Sullivan A P, Skog K, et al. Aerosol liquid water driven by anthropogenic nitrate: Implications for lifetimes of water-soluble organic gases and potential for secondary organic aerosol formation. Environ Sci Technol, 2014, 48: 11127-11136

16 Adams P J, Seinfeld J H, Koch D, et al. General circulation model assessment of direct radiative forcing by the sulfate-nitrate-ammonium-water inorganic aerosol system. J Geophys Res, 2001, 106: 1097-1111

17 Abbatt J P D, Lee A K Y, Thornton J A. Quantifying trace gas uptake to tropospheric aerosol: Recent advances and remaining challenges. Chem Soc Rev, 2012, 41: 6555-6581

18 Kolb C E, Cox R A, Abbatt J P D, et al. An overview of current issues in the uptake of atmospheric trace gases by aerosols and clouds. Atmos Chem Phys, 2010, 10: 10561-10605

19 Donaldson D J, Valsaraj K T. Adsorption and reaction of trace gas-phase organic compounds on atmospheric water film surfaces: A critical review. Environ Sci Technol, 2010, 44: 865-873

20 Zhao R. Aqueous-phase organic chemistry in the atmosphere. Doctor Dissertation. Toronto: University of Toronto, 2015

21 Gligorovski S, Strekowski R, Barbati S, et al. Environmental implications of hydroxyl radicals ('OH). Chem Rev, 2015, 115: 13051-13092

22 Park J H, Ivanov A V, Molina M J. Effect of relative humidity on OH uptake by surfaces of atmospheric importance. J Phys Chem A, 2008, 112: 6968-6977

23 Yu L, Smith J, Laskin A, et al. Chemical characterization of SOA formed from aqueous-phase reactions of phenols with the triplet excited state of carbonyl and hydroxyl radical. Atmos Chem Phys, 2014, 14: 13801-13816

24 Lim Y B, Tan Y, Turpin B J. Chemical insights, explicit chemistry, and yields of secondary organic aerosol from $\mathrm{OH}$ radical oxidation of methylglyoxal and glyoxal in the aqueous phase. Atmos Chem Phys, 2013, 13: 8651-8667

25 Slade J H, Knopf D A. Multiphase OH oxidation kinetics of organic aerosol: The role of particle phase state and relative humidity. Geophys Res Lett, 2014, 41: 5297-5306

26 Chan M N, Zhang H, Goldstein A H, et al. Role of water and phase in the heterogeneous oxidation of solid and aqueous succinic acid aerosol by hydroxyl radicals. J Phys Chem C, 2014, 118: 28978-28992

27 Davies J F, Wilson K R. Nanoscale interfacial gradients formed by the reactive uptake of OH radicals onto viscous aerosol surfaces. Chem Sci, 
2015, 6: 7020-7027

28 Lam H K, Shum S M, Davies J F, et al. Effects of inorganic salts on the heterogeneous OH oxidation of organic compounds: Insights from methylglutaric acid-ammonium sulfate. Atmos Chem Phys, 2019, 19: 9581-9593

$29 \mathrm{Xu} \mathrm{R}, \mathrm{Lam} \mathrm{H} \mathrm{K}$, Wilson K R, et al. Effect of inorganic-to-organic mass ratio on the heterogeneous OH reaction rates of erythritol: Implications for atmospheric chemical stability of 2-methyltetrols. Atmos Chem Phys, 2020, 20: 3879-3893

$30 \mathrm{Hu} \mathrm{W}$, Palm B B, Day D A, et al. Volatility and lifetime against OH heterogeneous reaction of ambient isoprene-epoxydiols-derived secondary organic aerosol (IEPOX-SOA). Atmos Chem Phys, 2016, 16: 11563-11580

31 Li Z, Smith K A, Cappa C D. Influence of relative humidity on the heterogeneous oxidation of secondary organic aerosol. Atmos Chem Phys, 2018, 18: 14585-14608

32 Liu S, Tsona N T, Zhang Q, et al. Influence of relative humidity on cyclohexene SOA formation from OH photooxidation. Chemosphere, 2019, 231: $478-486$

33 De Haan D O. Aqueous aerosol processing of glyoxal and methylglyoxal: Recent measurements of uptake coefficients, SOA production, and brown carbon formation. In: Hunt S W, Laskin A, Nizkorodov S A, eds. Multiphase Environmental Chemistry in the Atmosphere. Washington DC: American Chemical Society, 2018. 149-167

34 Hodzic A, Kasibhatla P S, Jo D S, et al. Rethinking the global secondary organic aerosol (SOA) budget: Stronger production, faster removal, shorter lifetime. Atmos Chem Phys, 2015, 16: 7917-7941

35 Curry L A, Tsui W G, McNeill V F. Technical note: Updated parameterization of the reactive uptake of glyoxal and methylglyoxal by atmospheric aerosols and cloud droplets. Atmos Chem Phys, 2018, 18: 9823-9830

36 De Haan D O, Jimenez N G, de Loera A, et al. Methylglyoxal uptake coefficients on aqueous aerosol surfaces. J Phys Chem A, 2018, 122: 48544860

37 Tilgner A, Bräuer P, Wolke R, et al. Modelling multiphase chemistry in deliquescent aerosols and clouds using CAPRAM3.0i. J Atmos Chem, 2013, 70: 221-256

38 Forni L, Bahnemann D, Hart E J. Mechanism of the hydroxide ion-initiated decomposition of ozone in aqueous solution. J Phys Chem, 1982, 86: $255-259$

39 Zellner R, Exner M, Herrmann H. Absolute $\mathrm{OH}$ quantum yields in the laser photolysis of nitrate, nitrite and dissolved $\mathrm{H}_{2} \mathrm{O}_{2}$ at 308 and $351 \mathrm{~nm}$ in the temperature range 278-353 K. J Atmos Chem, 1990, 10: 411-425

40 Arakaki T, Faust B C. Sources, sinks, and mechanisms of hydroxyl radical ( $\mathrm{OH}$ ) photoproduction and consumption in authentic acidic continental cloud waters from Whiteface Mountain, New York: The role of the Fe(r) (r = II, III) photochemical cycle. J Geophys Res, 1998, 103: 3487-3504

41 Tong H, Arangio A M, Lakey P S J, et al. Hydroxyl radicals from secondary organic aerosol decomposition in water. Atmos Chem Phys, 2016, 16: $1761-1771$

42 Zhao R, Lee A K Y, Wang C, et al. The role of water in organic aerosol multiphase chemistry: Focus on partitioning and reactivity. In: Barker J R, Steiner A L, Wallington T J, eds. Advances in Atmospheric Chemistry. Vol 1. London: World Scientific, 2016. 95-184

43 Arakaki T, Anastasio C, Kuroki Y, et al. A general scavenging rate constant for reaction of hydroxyl radical with organic carbon in atmospheric waters. Environ Sci Technol, 2013, 47: 8196-8203

44 Chameides W L. The photochemistry of a remote marine stratiform cloud. J Geophys Res, 1984, 89: 4739-4755

45 Ortiz-Montalvo D L, Lim Y B, Perri M J, et al. Volatility and yield of glycolaldehyde SOA formed through aqueous photochemistry and droplet evaporation. Aerosol Sci Technol, 2012, 46: 1002-1014

46 Tan Y, Lim Y B, Altieri K E, et al. Mechanisms leading to oligomers and SOA through aqueous photooxidation: insights from OH radical oxidation of acetic acid and methylglyoxal. Atmos Chem Phys, 2012, 12: 801-813

47 Sorooshian A, Ng N L, Chan A W, et al. Particulate organic acids and overall water-soluble aerosol composition measurements from the 2006 Gulf of Mexico Atmospheric Composition and Climate Study (GoMACCS). J Geophys Res-Atmos, 2007, 112: D13201

48 McNeill V F, Woo J L, Kim D D, et al. Aqueous-phase secondary organic aerosol and organosulfate formation in atmospheric aerosols: A modeling study. Environ Sci Technol, 2012, 46: 8075-8081

49 Herrmann H, Hoffmann D, Schaefer T, et al. Tropospheric aqueous-phase free-radical chemistry: Radical sources, spectra, reaction kinetics and prediction tools. ChemPhysChem, 2010, 11: 3796-3822

50 Schaefer T, Schindelka J, Hoffmann D, et al. Laboratory kinetic and mechanistic studies on the OH-initiated oxidation of acetone in aqueous solution. J Phys Chem A, 2012, 116: 6317-6326

51 Sorooshian A, Lu M L, Brechtel F J, et al. On the source of organic acid aerosol layers above clouds. Environ Sci Technol, 2007, 41: 4647-4654

52 Sun Y L, Zhang Q, Anastasio C, et al. Insights into secondary organic aerosol formed via aqueous-phase reactions of phenolic compounds based on high resolution mass spectrometry. Atmos Chem Phys, 2010, 10: 4809-4822

53 Smith J D, Sio V, Yu L, et al. Secondary organic aerosol production from aqueous reactions of atmospheric phenols with an organic triplet excited 
state. Environ Sci Technol, 2014, 48: 1049-1057

54 Guzman M I, Colussi A J, Hoffmann M R. Photoinduced oligomerization of aqueous pyruvic acid. J Phys Chem A, 2006, 110: 3619-3626

55 Renard P, Siekmann F, Gandolfo A, et al. Radical mechanisms of methyl vinyl ketone oligomerization through aqueous phase OH-oxidation: On the paradoxical role of dissolved molecular oxygen. Atmos Chem Phys, 2013, 13: 6473-6491

56 Perri M J, Lim Y B, Seitzinger S P, et al. Organosulfates from glycolaldehyde in aqueous aerosols and clouds: Laboratory studies. Atmos Environ, 2010, 44: 2658-2664

57 Schindelka J, Iinuma Y, Hoffmann D, et al. Sulfate radical-initiated formation of isoprene-derived organosulfates in atmospheric aerosols. Faraday Discuss, 2013, 165: 237-259

58 Schaefer T, van Pinxteren D, Herrmann H. Multiphase chemistry of glyoxal: Revised kinetics of the alkyl radical reaction with molecular oxygen and the reaction of glyoxal with $\mathrm{OH}, \mathrm{NO}_{3}$, and $\mathrm{SO}_{4}^{-}$in aqueous solution. Environ Sci Technol, 2015, 49: 343-350

59 Zhang X, Chen Z M, Zhao Y. Laboratory simulation for the aqueous OH-oxidation of methyl vinyl ketone and methacrolein: Significance to the in-cloud SOA production. Atmos Chem Phys, 2010, 10: 9551-9561

60 Schöne L, Schindelka J, Szeremeta E, et al. Atmospheric aqueous phase radical chemistry of the isoprene oxidation products methacrolein, methyl vinyl ketone, methacrylic acid and acrylic acid-kinetics and product studies. Phys Chem Chem Phys, 2014, 16: 6257-6272

61 Biswal J, Paul J, Naik D B, et al. Radiolytic degradation of 4-nitrophenol in aqueous solutions: Pulse and steady state radiolysis study. Radiat Phys Chem, 2013, 85: 161-166

62 Faust B C, Anastasio C, Allen J M, et al. Aqueous-phase photochemical formation of peroxides in authentic cloud and fog waters. Science, 1993, 260: $73-75$

63 Dalrymple R M, Carfagno A K, Sharpless C M. Correlations between dissolved organic matter optical properties and quantum yields of singlet oxygen and hydrogen peroxide. Environ Sci Technol, 2010, 44: 5824-5829

64 Kaur R, Hudson B M, Draper J, et al. Aqueous reactions of organic triplet excited states with atmospheric alkenes. Atmos Chem Phys, 2019, 19: $5021-5032$

65 Sareen N, Schwier A N, Shapiro E L, et al. Secondary organic material formed by methylglyoxal in aqueous aerosol mimics. Atmos Chem Phys, 2010, 10: 997-1016

66 Schwier A N, Sareen N, Mitroo D, et al. Glyoxal-methylglyoxal cross-reactions in secondary organic aerosol formation. Environ Sci Technol, 2010, 44: 6174-6182

67 Li Z, Schwier A N, Sareen N, et al. Reactive processing of formaldehyde and acetaldehyde in aqueous aerosol mimics: Surface tension depression and secondary organic products. Atmos Chem Phys, 2011, 11: 11617-11629

68 Holmes B J, Petrucci G A. Water-soluble oligomer formation from acid-catalyzed reactions of levoglucosan in proxies of atmospheric aqueous aerosols. Environ Sci Technol, 2006, 40: 4983-4989

69 Holmes B J, Petrucci G A. Oligomerization of levoglucosan by Fenton chemistry in proxies of biomass burning aerosols. J Atmos Chem, 2007, 58: $151-166$

70 Nozière B, Esteve W. Light-absorbing aldol condensation products in acidic aerosols: Spectra, kinetics, and contribution to the absorption index. Atmos Environ, 2007, 41: 1150-1163

71 Casale M T, Richman A R, Elrod M J, et al. Kinetics of acid-catalyzed aldol condensation reactions of aliphatic aldehydes. Atmos Environ, 2007, 41: $6212-6224$

72 De Haan D O, Corrigan A L, Smith K W, et al. Secondary organic aerosol-forming reactions of glyoxal with amino acids. Environ Sci Technol, 2009, 43: 2818-2824

73 Nozière B. Organic reactions increasing the absorption index of atmospheric sulfuric acid aerosols. Geophys Res Lett, 2005, 32: L03812

74 Noziere B, Dziedzic P, Cordova A, et al. Formation of secondary light-absorbing "fulvic-like" oligomers: A common process in aqueous and ionic atmospheric particles? Geophys Res Lett, 2007, 34: 1-5

75 Nguyen T B, Laskin A, Laskin J, et al. Brown carbon formation from ketoaldehydes of biogenic monoterpenes. Faraday Discuss, 2013, 165: 473494

76 Powelson M H, Espelien B M, Hawkins L N, et al. Brown carbon formation by aqueous-phase carbonyl compound reactions with amines and ammonium sulfate. Environ Sci Technol, 2014, 48: 985-993

77 Kampf C J, Jakob R, Hoffmann T. Identification and characterization of aging products in the glyoxal/ammonium sulfate system—Implications for light-absorbing material in atmospheric aerosols. Atmos Chem Phys, 2012, 12: 6323-6333

78 Drozd G T, McNeill V F. Organic matrix effects on the formation of light-absorbing compounds from $\alpha$-dicarbonyls in aqueous salt solution. Environ Sci-Process Impacts, 2014, 16: 741-747

79 Sedehi N, Takano H, Blasic VA, et al. Temperature- and pH-dependent aqueous-phase kinetics of the reactions of glyoxal and methylglyoxal with atmospheric amines and ammonium sulfate. Atmos Environ, 2013, 77: 656-663 
80 Gao S, Ng N L, Keywood M, et al. Particle phase acidity and oligomer formation in secondary organic aerosol. Environ Sci Technol, 2004, 38: $6582-6589$

81 Liggio J, Li S M, McLaren R. Heterogeneous reactions of glyoxal on particulate matter: Identification of acetals and sulfate esters. Environ Sci Technol, 2005, 39: 1532-1541

82 Surratt J D, Kroll J H, Kleindienst T E, et al. Evidence for organosulfates in secondary organic aerosol. Environ Sci Technol, 2007, 41: 517-527

83 Daumit K E, Carrasquillo A J, Hunter J F, et al. Laboratory studies of the aqueous-phase oxidation of polyols: Submicron particles vs. bulk aqueous solution. Atmos Chem Phys, 2014, 14: 10773-10784

84 Huang L, Cochran R E, Coddens E M, et al. Formation of organosulfur compounds through transition metal ion-catalyzed aqueous phase reactions. Environ Sci Technol Lett, 2018, 5: 315-321

85 Guo H, Xu L, Bougiatioti A, et al. Fine-particle water and pH in the southeastern United States. Atmos Chem Phys, 2014, 15: 5211-5228

86 Weber R J, Guo H, Russell A G, et al. High aerosol acidity despite declining atmospheric sulfate concentrations over the past 15 years. Nat Geosci, 2016, 9: 282-285

87 Huang L, Coddens E M, Grassian V H. Formation of organosulfur compounds from aqueous phase reactions of S(IV) with methacrolein and methyl vinyl ketone in the presence of transition metal ions. ACS Earth Space Chem, 2019, 3: 1749-1755

88 Richards-Henderson N K, Pham A T, Kirk B B, et al. Secondary organic aerosol from aqueous reactions of green leaf volatiles with organic triplet excited states and singlet molecular oxygen. Environ Sci Technol, 2015, 49: 268-276

89 Huang D D, Zhang Q, Cheung H H Y, et al. Formation and evolution of aqSOA from aqueous-phase reactions of phenolic carbonyls: Comparison between ammonium sulfate and ammonium nitrate solutions. Environ Sci Technol, 2018, 52: 9215-9224

90 Kirkland J R, Lim Y B, Tan Y, et al. Glyoxal secondary organic aerosol chemistry: Effects of dilute nitrate and ammonium and support for organic radical-radical oligomer formation. Environ Chem, 2013, 10: 158-166

91 Bruns E A, El Haddad I, Keller A, et al. Inter-comparison of laboratory smog chamber and flow reactor systems on organic aerosol yield and composition. Atmos Meas Tech, 2015, 8: 2315-2332

$92 \mathrm{Ng} \mathrm{N} \mathrm{L}$, Canagaratna M R, Zhang Q, et al. Organic aerosol components observed in Northern Hemispheric datasets from Aerosol Mass Spectrometry. Atmos Chem Phys, 2010, 10: 4625-4641

93 Alfarra M R, Paulsen D, Gysel M, et al. A mass spectrometric study of secondary organic aerosols formed from the photooxidation of anthropogenic and biogenic precursors in a reaction chamber. Atmos Chem Phys, 2006, 6: 5279-5293

94 Iinuma Y, Müller C, Böge O, et al. The formation of organic sulfate esters in the limonene ozonolysis secondary organic aerosol (SOA) under acidic conditions. Atmos Environ, 2007, 41: 5571-5583

95 Wang Y, Hu M, Guo S, et al. The secondary formation of organosulfates under interactions between biogenic emissions and anthropogenic pollutants in summer in Beijing. Atmos Chem Phys, 2018, 18: 10693-10713

96 Cai D, Wang X, Chen J, et al. Molecular characterization of organosulfates in highly polluted atmosphere using ultra-high-resolution mass spectrometry. J Geophys Res-Atmos, 2020, 125: e2019JD032253

97 Schmitt-Kopplin P, Gelencser A, Dabek-Zlotorzynska E, et al. Analysis of the unresolved organic fraction in atmospheric aerosols with ultrahighresolution mass spectrometry and nuclear magnetic resonance spectroscopy: Organosulfates as photochemical smog constituents. Anal Chem, 2010, 82: 8017-8026

98 Xie Q, Li Y, Yue S, et al. Increase of high molecular weight organosulfate with intensifying urban air pollution in the megacity Beijing. J Geophys Res-Atmos, 2020, 125: e2019JD032200

99 Lopez-Hilfiker F D, Mohr C, Ehn M, et al. A novel method for online analysis of gas and particle composition: Description and evaluation of a Filter Inlet for Gases and AEROsols (FIGAERO). Atmos Meas Tech, 2013, 7: 983-1001

100 Le Breton M, Wang Y, Hallquist $\AA$ M, et al. Online gas- and particle-phase measurements of organosulfates, organosulfonates and nitrooxy organosulfates in Beijing utilizing a FIGAERO ToF-CIMS. Atmos Chem Phys, 2017, 18: 10355-10371

101 Lopez-Hilfiker F D, Pospisilova V, Huang W, et al. An extractive electrospray ionization time-of-flight mass spectrometer (EESI-TOF) for online measurement of atmospheric aerosol particles. Atmos Meas Tech, 2019, 12: 4867-4886

102 Doezema L A, Longin T, Cody W, et al. Analysis of secondary organic aerosols in air using extractive electrospray ionization mass spectrometry (EESI-MS). RSC Adv, 2012, 2: 2930-2938

103 Zhao R, Aljawhary D, Lee A K Y, et al. Rapid aqueous-phase photooxidation of dimers in the $\alpha$-pinene secondary organic aerosol. Environ Sci Technol Lett, 2017, 4: 205-210

104 Gallimore P J, Giorio C, Mahon B M, et al. Online molecular characterisation of organic aerosols in an atmospheric chamber using extractive electrospray ionisation mass spectrometry. Atmos Chem Phys, 2017, 17: 14485-14500

$105 \mathrm{Xu} \mathrm{W}$, Han T, Du W, et al. Effects of aqueous-phase and photochemical processing on secondary organic aerosol formation and evolution in Beijing, China. Environ Sci Technol, 2017, 51: 762-770 
$106 \mathrm{Xu} \mathrm{L}$, Guo H, Boyd C M, et al. Effects of anthropogenic emissions on aerosol formation from isoprene and monoterpenes in the southeastern United States. Proc Natl Acad Sci USA, 2015, 112: 37-42

107 Gilardoni S, Massoli P, Paglione M, et al. Direct observation of aqueous secondary organic aerosol from biomass-burning emissions. Proc Natl Acad Sci USA, 2016, 113: 10013-10018

108 Waxman E M, Dzepina K, Ervens B, et al. Secondary organic aerosol formation from semi- and intermediate-volatility organic compounds and glyoxal: Relevance of O/C as a tracer for aqueous multiphase chemistry. Geophys Res Lett, 2013, 40: 978-982

109 Knote C, Hodzic A, Jimenez J L, et al. Simulation of semi-explicit mechanisms of SOA formation from glyoxal in aerosol in a 3-D model. Atmos Chem Phys, 2014, 14: 6213-6239

110 Pye H O T, Pinder R W, Piletic I R, et al. Epoxide pathways improve model predictions of isoprene markers and reveal key role of acidity in aerosol formation. Environ Sci Technol, 2013, 47: 11056-11064

111 Woo J L, McNeill V F. simplegamma v1.0 - A reduced model of secondary organic aerosol formation in the aqueous aerosol phase (aaSOA). Geosci Model Dev, 2015, 8: 1821-1829

112 Song S, Gao M, Xu W, et al. Possible heterogeneous chemistry of hydroxymethanesulfonate (HMS) in northern China winter haze. Atmos Chem Phys, 2019, 19: 1357-1371

113 Ma T, Furutani H, Duan F, et al. Contribution of hydroxymethanesulfonate (HMS) to severe winter haze in the North China Plain. Atmos Chem Phys, 2020, 20: 5887-5897

\section{补充材料}

表S1 OH自由基在不同类型气溶胶上的摄取系数

表S2 甲基乙二醛在不同类型气溶胶上的摄取系数

本文以上补充材料见网络版csb.scichina.com. 补充材料为作者提供的原始数据, 作者对其学术质量和内容负责. 


\title{
Formation mechanism of secondary organic aerosol in aerosol liquid water: A review
}

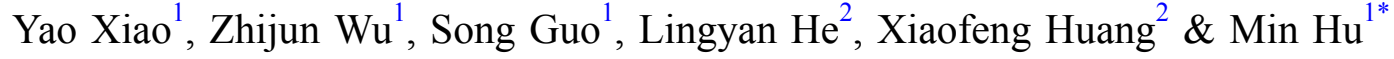 \\ ${ }^{1}$ State Key Joint Laboratory of Environmental Simulation and Pollution Control, College of Environmental Sciences and Engineering, Peking Univer- \\ sity, Beijing 100871, China: \\ ${ }^{2}$ Key Laboratory of Urban Habitat Environmental Science and Technology, School of Environment and Energy, Peking University Shenzhen Graduate \\ School, Shenzhen 518055, China \\ * Corresponding author, E-mail: minhu@pku.edu.cn
}

Secondary organic aerosol (SOA) is the main component of $\mathrm{PM}_{2.5}$, with great impact on regional air quality and global climate. The traditional view that SOA forms through the partitioning of photochemical processing involving volatile organic compounds (VOCs) cannot fully explain measured SOA concentrations. It has been increasing recognized that SOA can form through aqueous reactions in recent years. Besides cloud/fog aqueous chemistry, aqueous SOA (aqSOA) formation in aerosol liquid water has become one of the frontier scientific problems of atmospheric chemistry. AqSOA precursors enter into aqueous phase through uptake to wet aerosol particles, participate in reactions inside aerosol particles, and then form aqSOA such as organic sulfur compounds and organic nitrogen compounds. This paper provides an overview of the uptake of aqSOA precursors, aqSOA formation mechanism and current research methods of aqSOA. AqSOA precursors include atmospheric oxidants $\left(\mathrm{OH}, \mathrm{HO}_{2}, \mathrm{O}_{3}\right.$, etc.), anthropogenic and biogenic VOCs and related gasphase oxidation products. Aerosol liquid water can influence the uptake of aqSOA precursors on wet aerosols, but related researches are limited. $\mathrm{OH}$ uptake coefficient $\left(\gamma_{\mathrm{OH}}\right)$ on different kinds of aerosols varies from 0.02 to 2.41, depending on chemical composition of aerosols and relative humidity. For the uptake of VOCs, take methylglyoxal as an example, measured and theoretical methylglyoxal uptake coefficient $\left(\gamma_{\mathrm{MGLY}}\right)$ differ by 4 to 5 orders of magnitude. Aerosol liquid water may change ionic strength, diffusion limitation and viscosity of wet aerosols, but how these affect the uptake process of aqSOA precursors remain poorly understood. Based on previous analyses of aqueous chemistry, aqueous-phase reactions can be divided into radical reactions and non-radical reactions. Aqueous-phase radical reactions resemble gasphase reactions in general. However, there are also $\mathrm{OH}$ radical reactions unique to the aqueous phase: efficient conversion of aldehydes to carboxylic acids, rapid $\mathrm{OH}$ oxidation of carboxylate, and radical induced oligomerization. Recent studies also pay increasing attention to the role of other oxidants in the aqueous radical chemistry, like singlet oxygen, peroxyl radicals, peroxides, molecular oxygen $\left(\mathrm{O}_{2}{ }^{*}\right)$, and triplet excited states of organic compounds $\left({ }^{3} \mathrm{C}^{*}\right)$. Non-radical reactions include hemiacetal formation, aldol condensation, imine formation and other types of reactions (anhydride formation, organosulfate formation, etc.). Most non-radical reactions lead to the formation of high molecular weight compounds. Although a lot of investigations have been taken to explore aqSOA formation mechanism, the majority are laboratory studies, because of the limit of technology in field measurements. Laboratory simulation includes bulk solution simulation and reaction chamber experiments. Bulk solution cannot simulate typical ambient wet aerosols well, so nowadays reaction chamber is used more widely. There are two types of reaction chambers: smog chamber and flow tube, differing in the volume of reaction chamber and simulated atmospheric oxidation timescale. However, the appropriate application of these laboratory results into field observations and model framework needs further efforts. Moreover, one key factor that has enabled great progress in aqSOA chemistry studies is the development of mass-spectrometric methods, mainly including electrospray ionization-mass spectrometry (ESI-MS), Fourier transform ion cyclotron resonance electrospray ionization mass spectrometry (FTICR-MS), chemical ionization mass spectrometry (CIMS) and extractive electrospray ionizationmass spectrometry (EESI-MS). These techniques can realize accurate molecular level identification of complex compounds. But quantification remains a thorny issue, owing to the absence of available authentic standards. Finally, possible future directions regarding aqSOA chemistry studies are discussed.

aqueous secondary organic aerosol, precursor, uptake, aqueous-phase reaction, formation mechanism, measurement technique

doi: 10.1360/TB-2020-0713 